\title{
Coherent control of an opsin in living brain tissue
}

\author{
Kush Paul ${ }^{1,2 \uparrow}$, Parijat Sengupta ${ }^{1,2,3 \uparrow}{ }^{2}$, Eugene D. Ark ${ }^{1,2,4}$, Haohua Tu${ }^{1}$, Youbo Zhao ${ }^{1}$ \\ and Stephen A. Boppart ${ }^{1,2,3,4 \star}$
}

Retinal-based opsins are light-sensitive proteins. The photoisomerization reaction of these proteins has been studied outside cellular environments using ultrashort tailored light pulses $^{1-5}$. However, how living cell functions can be modulated via opsins by modifying fundamental nonlinear optical properties of light interacting with the retinal chromophore has remained largely unexplored. We report the use of chirped ultrashort near-infrared pulses to modulate light-evoked ionic current from Channelrhodopsin-2 (ChR2) in brain tissue, and consequently the firing pattern of neurons, by manipulating the phase of the spectral components of the light. These results confirm that quantum coherence of the retinal-based protein system, even in a living neuron, can influence its current output, and open up the possibilities of using designer-tailored pulses for controlling molecular dynamics of opsins in living tissue to selectively enhance or suppress neuronal function for adaptive feedback-loop applications in the future.

Controlling cell functions with light has been achieved in recent years using natural and synthetic photosensitive proteins ${ }^{6,7}$, and retinal-based opsins, such as $\mathrm{ChR} 2$, are at the centre of this field. Usually mutant opsins are genetically engineered to improve cell activation at a specific wavelength or to make the protein predominantly absorb a different colour of light; parameters governing nonlinear light properties, such as phase and amplitude of an ultrashort light pulse, are seldom tweaked to generate a different response from an opsin. Even though two-photon excitation has been used to achieve better spatial specificity and deeper penetration in scattering brain tissue ${ }^{8}$, for three-dimensional imaging of neuronal activity with wide-field temporal focusing ${ }^{9}$, and for optogenetic stimulation using temporally focused two-photon light pulses ${ }^{10,11}$, there have been far fewer studies that have explored the effects of the phase structure of the stimulation pulse on absorption by retinal. In fact, no reported study to date has used quantum coherent control to alter the current output from opsins in neurons.

Light-induced isomerization of the retinal chromophore from all-trans to 13-cis is the first event in the activation of ChR2 (ref. 12). (See Schneider et al. ${ }^{13}$ and references within for a review of ChR2 biophysics.) This photoisomerization is ultrafast, taking place on the femtosecond $\left(10^{-15} \mathrm{~s}\right)$ to picosecond $\left(10^{-12} \mathrm{~s}\right)$ timescale $\mathrm{e}^{5,14-17}$. At these fast timescales, quantum coherence effects in the lightmatter interaction can alter the population distribution of the quantum states, and hence the outcome of the reaction ${ }^{18}$. This can be tested with spectrally and temporally tailored ultrafast light pulses $^{19}$ where spectral modulation arranges different components of the wave packets in the ground and excited state potential surfaces, while temporal modulation organizes their relative phases, allowing control of wave packet propagation ${ }^{16}$. The group velocity delay (GVD) $\tau(\omega)$ of a given frequency component of the field is related to the phase via $\tau(\omega)=\delta \varphi(\omega) / \delta \omega$ (ref. 20). When $\tau(\omega)$ has no quadratic or higher-order dependence on frequency, the pulse is transform-limited (TL). These higher-order phase terms (see Methods) are known as chirps. The phase structure of the excitation pulse can be modulated using linear chirp (the secondorder phase term) where the frequency is a linear function of time and the chirp sign determines the temporal ordering of the pulse frequency components. The ordering of the frequency components in time causes lengthening of a transform-limited ultrafast pulse. When the chirp is positive ( $\mathrm{PCH})$, the low-frequency components are in the leading edge of the pulse reaching the sample before the high-frequency components, whereas for a negative chirp ( $\mathrm{NCH})$ the order is reversed.

For two-photon excitation of ChR2, ultrashort near-infrared (NIR) pulses were modulated using a pulse-shaper unit (Supplementary Fig. 1 and Methods) where the spectral contents of the pulse were spatially dispersed, modulated independently, and then recombined to create a complex tailored pulse ${ }^{21}$ for experiments. Phase-uncompensated output pulses from a photonic crystal fibre (PCF) ( $\tau=250 \mathrm{fs}$ ) were compressed to $\sim 30 \mathrm{fs}$ using the pulse shaper by aligning the phases of all spectral components (that is, coefficient of the quadratic term, $a 2=0$ ) to generate phase-compensated TL NIR pulses. Then, different linear chirps were introduced in the pulse by systematically varying $a 2$ in the spectral phase function. As rhodopsin shows a small wavelengthdependence of cis-trans isomerization photoreaction quantum yield $^{22,23}$, for designing pure coherent control experiments, only the phase was modulated, keeping the overlap between the ChR2 absorption spectrum and the laser spectrum unchanged. For a channel protein like ChR2 in its cellular environment, interference caused by thermal motion and random molecular fluctuations along many degrees of freedom can act to scramble a phase relationship within an optical pulse. Additionally, whether modulation of phases could play a role in directing biological functions for such a highly evolved complex membrane protein remains unclear. Therefore, we measured light-evoked ionic currents for all experiments as the output from neurons, which should be proportional to the ChR2 isomerization efficiency at different linear chirp, to emphasize that the quantum coherence effects in ChR2 were not merely transient, but actually were able to modulate biological function.

Layer V neurons from $300-\mu \mathrm{m}$-thick coronal brain slices obtained from ChR2-YFP mice were used for patch-clamp electrophysiology. First, a patch-clamped neuron was stimulated via one-photon absorption (1PA) of ChR2 with a $470 \mathrm{~nm}$ light-emitting diode (LED) to verify if the cell was responsive to light stimulation (Supplementary Fig. 2). For cells with substantial peak current, the

\footnotetext{
${ }^{1}$ Beckman Institute for Advanced Science and Technology, University of Illinois at Urbana-Champaign, Urbana, Illinois 61801, USA. ${ }^{2}$ Neuroscience Program, University of Illinois at Urbana-Champaign, Urbana, Illinois 61801, USA. ${ }^{3}$ Department of Bioengineering, University of Illinois at Urbana-Champaign, Urbana, Illinois 61801, USA. ${ }^{4}$ Department of Electrical and Computer Engineering, University of Illinois at Urbana-Champaign, Urbana, Illinois 61801, USA. †These authors contributed equally to this work. ${ }^{\star}$ e-mail: boppart@illinois.edu
} 
a

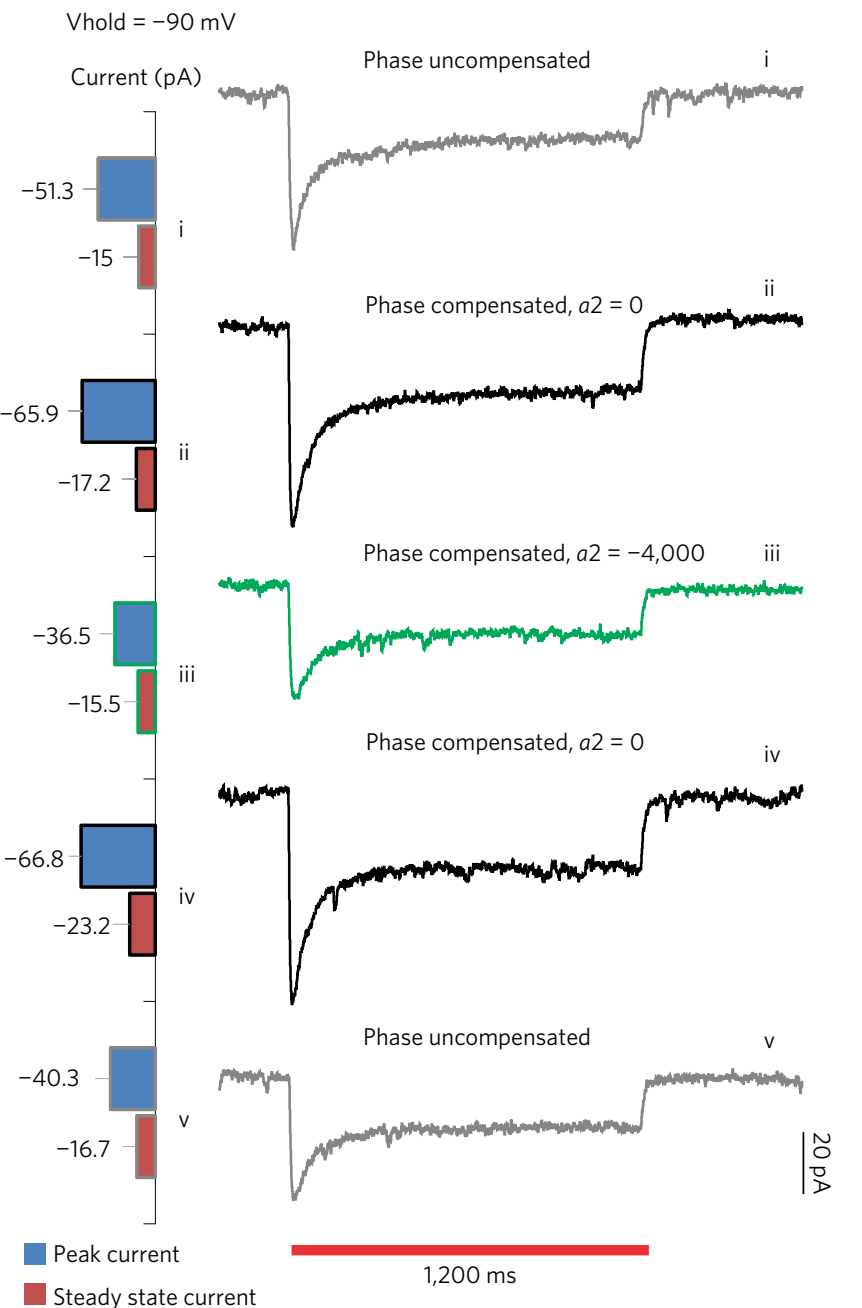

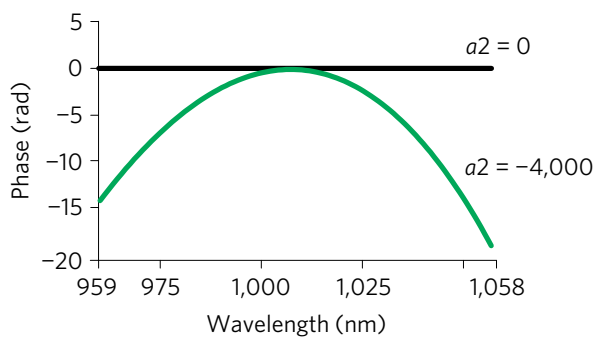

c

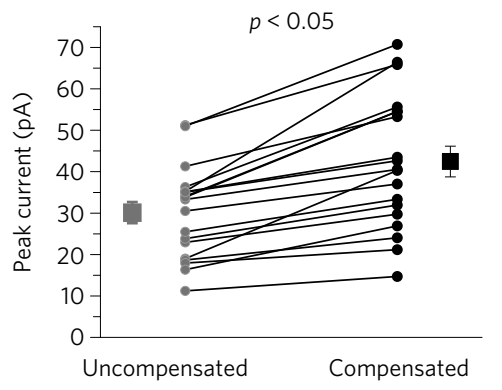

d

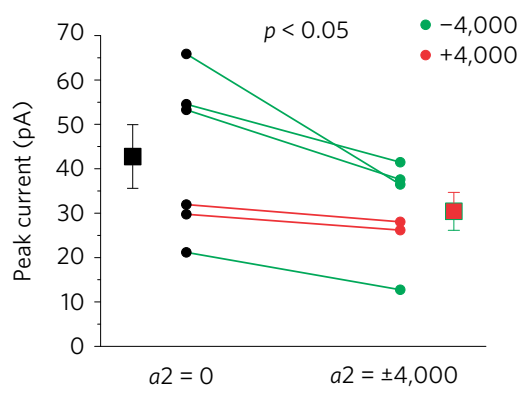

Figure 1 | Modulation of current output from ChR2-expressing neurons with tailored ultrashort pulsed light. a, Consecutive traces from a layer $V$ pyramidal neuron show modulation of the peak amplitude (i, ii, iii) and recovery (iv, v) of ChR2 current due to phase compensation and linear chirp. b. Phase envelope of spectral components for $a 2=0$ and $a 2=-4,000$. See also Supplementary Fig. 4. c, Population data of neurons show significant increase in the peak ChR2 current amplitude due to phase compensation. Uncompensated: $30 \pm 3$ pA. Compensated: $43 \pm 4 p A, n=19, p<0.005$. d, A significant decrease in the peak amplitude is observed with the addition of linear chirp of $a 2= \pm 4,000 \mathrm{fs}^{2} ; a 2=0 \mathrm{fs}^{2}: 43 \pm 7 \mathrm{pA}, a 2= \pm 4,000 \mathrm{fs}^{2}$ : $30 \pm 4 \mathrm{pA}(n=6, p<0.05)$. Data is plotted as mean \pm s.e.m. All currents are negative inward currents.

light source was then switched to the output from the pulse shaper, which delivered the tailored light pulses for two-photon absorption (TPA) by ChR2. The response from the neuron to $1,200 \mathrm{~ms}$ NIR stimulation was measured with phase-uncompensated pulses, compressed TL pulses, and chirped $\left(a 2=-4,000 \mathrm{fs}^{2}\right)$ pulses (Fig. 1). The peak current evoked with TPA of TL pulses (average power at focus: $10 \mathrm{~kW} \mathrm{~mm}{ }^{-2}$; fluence: $5.4-8 \times 10^{16}$ photons $\mathrm{cm}^{-2}$ pulse $^{-1}$ ) was about $8 \%(n=13$ neurons) of the $1 \mathrm{PA}$ current (at excitation power $0.9 \mathrm{~mW} \mathrm{~mm}^{-2}$ ). No apparent thermal damage to the neurons was observed with $1,200 \mathrm{~ms}$ NIR laser exposure at this power (Supplementary Fig. 3).

Five consecutive current traces of the response of a pyramidal neuron under these three conditions (Fig. 1a) show that pulse compression (phase compensation) substantially increased the peak current whereas linear chirp reduced it. The phase of the light pulse at different spectral components for $a 2=0 \mathrm{fs}^{2}$ and $-4,000 \mathrm{fs}^{2}$ is shown in Fig. 1b. The population data for peak current (Fig. 1c) showed that pulse compression to a TL pulse produced $41 \%$ more current output, which is significantly larger (Uncompensated: $-30 \pm 3 \mathrm{pA}$, Compensated: $-43 \pm 4 \mathrm{pA}$, $n=19, p<0.005)$. Phase compensation also modified steady-state current and activation rates (Supplementary Fig. 4). Linear chirp with $a 2= \pm 4,000 \mathrm{fs}^{2}$ tested in a subset of these neurons (Fig. 1d) showed a significant $29 \%$ decrease in the peak current amplitude from $-43 \pm 7 \mathrm{pA}$ to $-30 \pm 4 \mathrm{pA}(n=6, p<0.05)$, which could subsequently be reversed by its removal.

The probability of TPA of ChR2 per laser pulse depends on its TPA cross-section $\left(\sigma_{2}\right)$, peak power $\left(P_{\text {peak }}\right)$ of the pulse, and pulse width $\left(\tau_{\mathrm{p}}\right)$ (equation (5)). As we only modulated the temporal relationship between frequency components by introducing linear chirp, the spectral overlap between the ChR2 activation spectrum and the laser pulse spectrum remained largely unaltered. Therefore, the observed effect is not due to a change in the overlap integral. During phase compensation, $\tau_{\mathrm{p}}$ was shortened from $250 \mathrm{fs}$ to $30 \mathrm{fs}$, which enhanced TPA, and hence, the ensuing current (Fig. 1c). Similarly, introduction of chirp $\left(a 2=+4,000\right.$ or $\left.-4,000 \mathrm{fs}^{2}\right)$ broadened the pulse (equation (2) in the Methods) and reduced neuronal current output (Fig. 1d). These effects can be explained using an effective TPA cross-section argument that is related to $P_{\text {peak }}$. However, the peak current behaves somewhat differently from a 'normal' TPA process. A closer look into current output with $\mathrm{PCH}$ and $\mathrm{NCH}$ revealed a more intriguing outcome.

In general, a test for coherence, like other properties of waves, requires demonstration of both constructive and destructive 
a

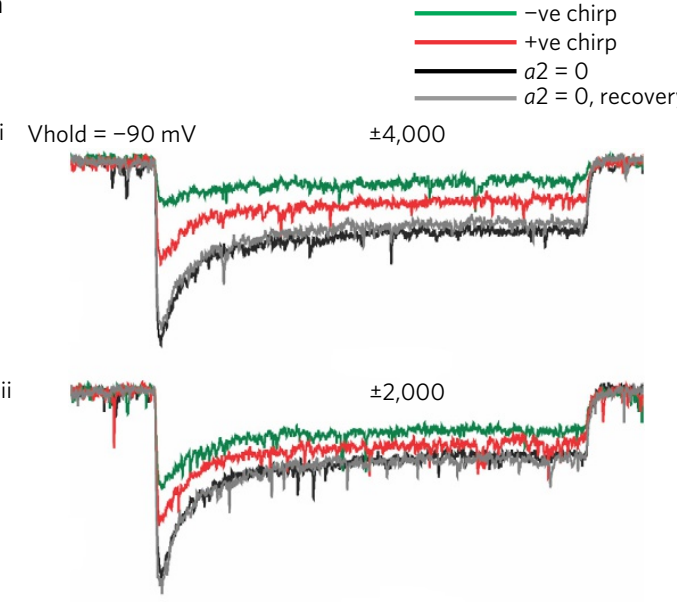

ii

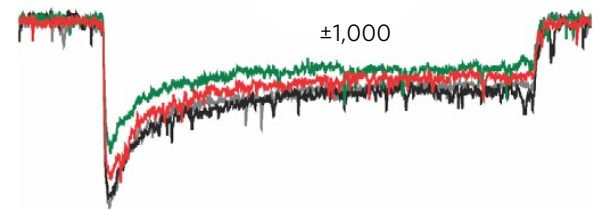

iv

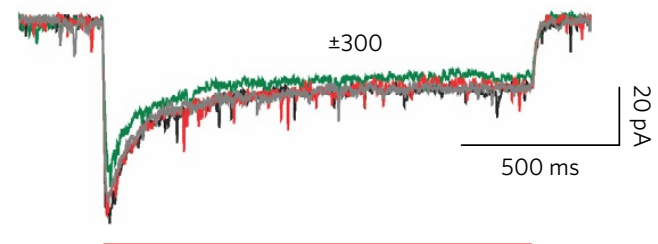

$1,200 \mathrm{~ms}$

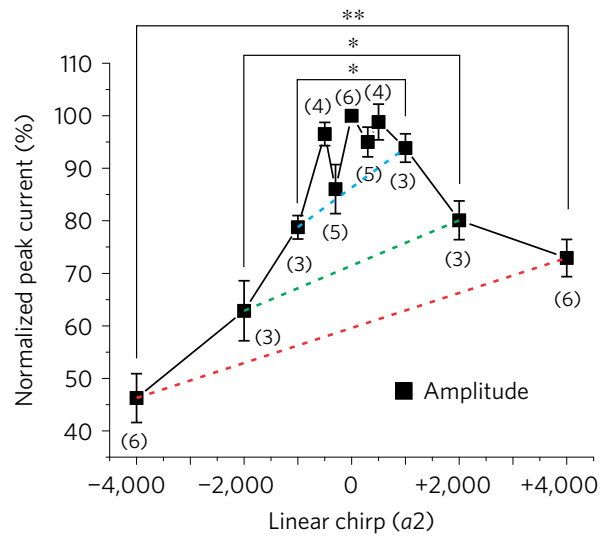

b
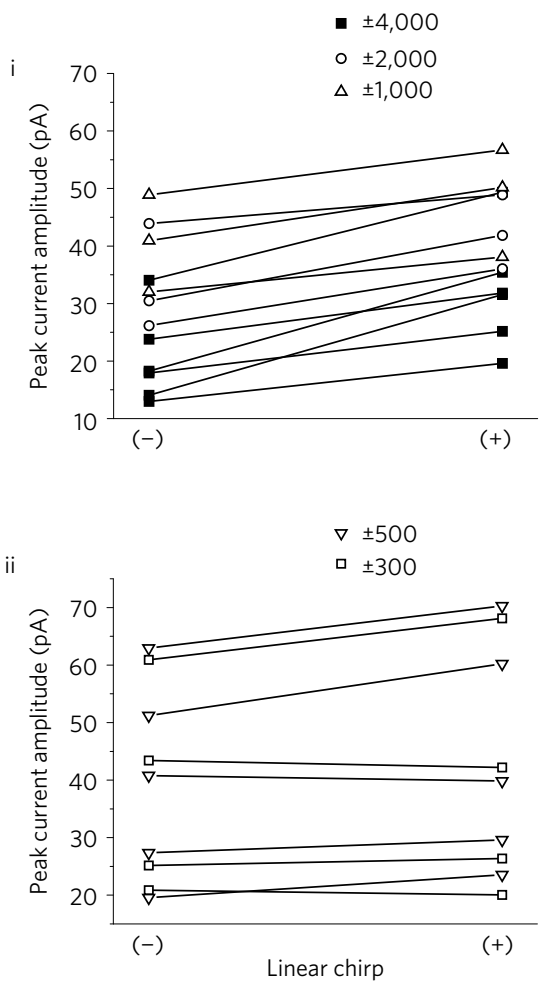

d

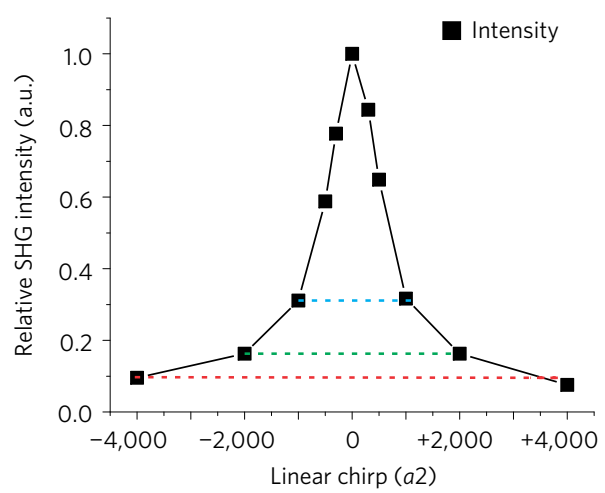

Figure 2 | The light-evoked current output from ChR2-expressing neurons can be altered by modulating the phase structure of the ultrashort light pulse. a, (i) Consecutive traces from a voltage clamped neuron show response to $a 2=0 \mathrm{fs}^{2}$ (black), $a 2=-4,000 \mathrm{fs}^{2}$ (green), a2 $=+4,000 \mathrm{fs}^{2}$ (red) and $a 2=0 \mathrm{fs}^{2}$ recovery (grey), in that order. (ii, iii, iv) Similar to above, traces shown with $a 2$ (in $\mathrm{fs}^{2}$ ) $= \pm 2,000, \pm 1,000$ and \pm 300 from the same neuron. b. (i) Individual peak current amplitudes for corresponding negative and positive linear chirp for $a 2$ (in $\mathrm{fs}^{2}$ ) $= \pm 4,000, \pm 2,000$ and $\pm 1,000$. (ii) Individual peak current amplitudes for corresponding negative and positive linear chirp for $a 2$ (in fs ${ }^{2}$ ) $= \pm 500$ and \pm 300 . c, Summary of normalized peak current amplitudes for negative and positive linear chirps. All currents were normalized to the phase-compensated condition $\left(a 2=0 \mathrm{fs}^{2}\right)$. The numbers in parentheses refer to the number of neurons the currents were measured in at each linear chirp. Asterisks represent statistical significance: ${ }^{*} p<0.05$, ${ }^{* *} p<0.005$. d, SHG intensities at different linear chirp: $a 2$ (in $\left.\mathrm{fs}^{2}\right)= \pm 4,000, \pm 2,000$ and $\pm 1,000$. The SHG intensity at $a 2=4,000 \mathrm{fs}^{2}$ is almost identical for PCH (0.075) and NCH (0.091); however, the peak current amplitudes in c are significantly different. See also Supplementary Figs 5 and 7. Data is plotted as mean \pm s.e.m. All currents are negative inward currents.

interference that are expected to change an observable differently $y^{2,3}$. Therefore, the effects of $\mathrm{PCH}$ and $\mathrm{NCH}$ pulses on whole-cell ionic currents were investigated more exhaustively (Fig. 2) to test this critical point. $\mathrm{PCH}$ and $\mathrm{NCH}$ pulses with the same coefficient, $a 2$, have identical peak intensity and temporal pulse width but vary in the instantaneous frequency. For current measurement, $a 2$ was varied in the following order (Fig. 2a): a2 (in $\left.\mathrm{fs}^{2}\right)=0,-4,000,+4,000,0$ (recovery). This was repeated for all $a 2$ values (Fig. $2 \mathrm{a}-\mathrm{c}$ ). In a representative neuron (Fig. 2a) the peak current at $a 2=0$ was $-54.5 \mathrm{pA}$ at the start of the trials. In successive trials with $a 2=-4,000 \mathrm{fs}^{2}$ and $a 2=+4,000 \mathrm{fs}^{2}$, the corresponding peak current values were $-14.1 \mathrm{pA}$ and $-31.6 \mathrm{pA}$, respectively. The light-evoked peak current values for other $a 2$ values were as follows: $-30.5 \mathrm{pA}\left(-2,000 \mathrm{fs}^{2}\right)$ and 

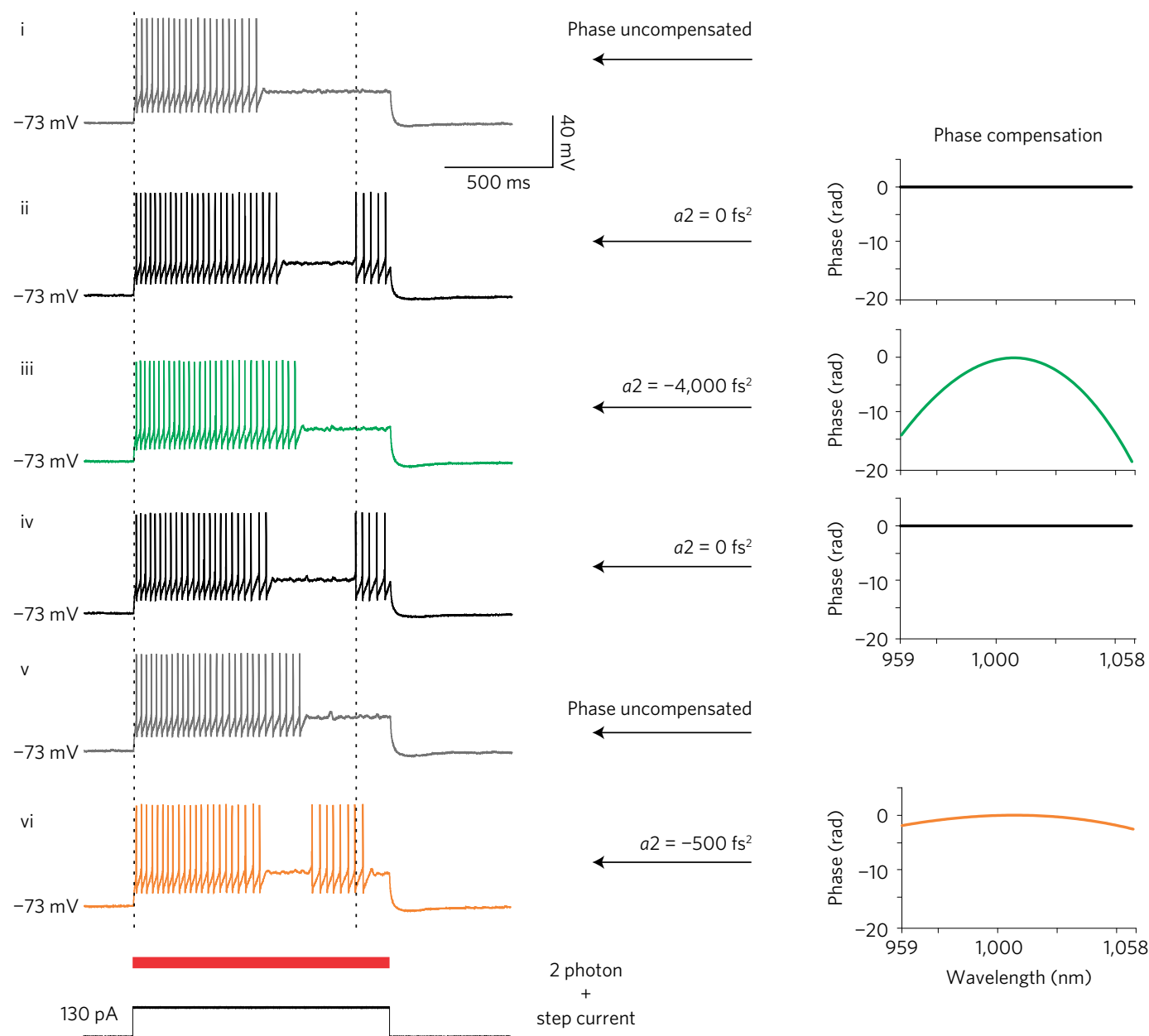

Figure 3 | Modulation of phase structure altered the spiking pattern of a fast-spiking (FS) interneuron. Spike response of a FS cortical interneuron is modulated by phase of the spectrally broadened light pulse, seen here in consecutive traces (i-vi). The spectrally shaped light pulse with spectral phase compensation and linear chirp was superimposed on a fixed step current pulse (130 pA) so that the membrane potential was just below the spike threshold. Notice that a specific linear chirp value reliably produces a specific spiking pattern (indicated with different colours). The dashed line is used to indicate that conditions (ii) and (iv) show the same pattern. The flat potential region indicates an elevated membrane potential region (threshold potential), and a region where no action potentials (or spikes) were generated. The pulse duration for each pulse was $\leq 350 \times 10^{-15} \mathrm{~s}$ (Supplementary Fig. 7). The laser light was 'on' (or consider that the shutter was open) for 1,200 ms, which is denoted by the red bar in this figure.

$-41.8 \mathrm{pA}\left(+2,000 \mathrm{fs}^{2}\right) ;-40.9 \mathrm{pA} \quad\left(-1000 \mathrm{fs}^{2}\right)$ and $-50.2 \mathrm{pA}$ $\left(+1,000 \mathrm{fs}^{2}\right) ;-51.2 \mathrm{pA}\left(-300 \mathrm{fs}^{2}\right)$ and $-60.2 \mathrm{pA}\left(+300 \mathrm{fs}^{2}\right)$. We observed significantly larger ionic currents with $\mathrm{PCH}$ compared to $\mathrm{NCH}$ for $a 2$ (in $\mathrm{fs}^{2}$ ) $=4,000,2,000$ and 1,000 (Fig. $2 \mathrm{~b}, \mathrm{c}$ ). The trend continued for lower chirp values, but the differences were not statistically significant (Fig. 2b,c). Phase modulation also altered steady-state current and the initial activation slope (Supplementary Fig. 5). Evoked-current outputs with $a 2\left(\right.$ in $\left.\mathrm{fs}^{2}\right)=0,+4,000$ and $-4,000$ were measured at different average laser power and indicated that experiments were performed close to saturation power but not at saturation (Supplementary Fig. 6).

Moreover, pulse intensities measured using second harmonic generation (SHG) with a fraction of the $\mathrm{PCH}$ and $\mathrm{NCH}$ excitation light (see Methods) were almost identical at various $a 2$ values (Fig. 2d), but the light-evoked ionic currents were significantly lower for $\mathrm{NCH}$ compared to $\mathrm{PCH}$ (Fig. 2c). This confirms that there was nothing systematic in the laser pulse, other than phase, causing the enhancements in the TPA of all-trans retinal with the PCH pulse. This is a coherent result as it is achieved through temporal ordering of the pulse frequency components.

An average of theoretically calculated and experimentally estimated TPA using equivalent non-chirped pulses is presented in Supplementary Fig. $7 \mathrm{~b}$ for comparison with measured current values at different $\mathrm{PCH}$ and $\mathrm{NCH}$ pulses (see Methods). Additionally, our data demonstrated that, irrespective of the sign, the evoked peak current with linear chirp was always higher than what would be the theoretical TPA-only current (Supplementary Fig. 7b). This is indicative of a situation where tailoring of the laser pulse could enhance the outcome from a biological system, when compared to an equivalent Gaussian pulse of same duration and number of photons (see Supplementary Discussion 1).

Next, we tested if the spiking pattern of a neuron could be modulated using chirped pulses, and investigated fast-spiking (FS) interneurons. These neurons have a very linear spike frequency response pattern to increasing current or light intensities, and are sensitive to small changes in membrane potential. An input step current pulse was injected through the recording micropipette synchronously with the spectrally shaped light pulse (Fig. 3). Consecutive traces of spike output from a FS interneuron show different spike patterns with $a 2$ (in $\left.\mathrm{fs}^{2}\right)=0,-4,000$, and 0 (recovery). A different linear chirp, $a 2=-500 \mathrm{fs}^{2}$, produced a spiking pattern that was different from both the $a 2=0 \mathrm{fs}^{2}$ phasecompensated and the $a 2=-4,000 \mathrm{fs}^{2}$ phase conditions (Fig. 3). Most published work in optogenetics used short (millisecond) illumination pulses at a desired frequency to spike-tune a neuron. In contrast, these data offer the first proof for the concept that 
quantum coherent control with tailored femtosecond pulses can vary the spike output, and therefore the functional state of the neuron (see Supplementary Discussion 2).

The photoisomerization reaction of retinal has been investigated theoretically and experimentally $y^{5,14,24-30}$ in the past, including the possibility of manipulating this reaction by tailored pulses ${ }^{1-5,30}$, but these experiments were not performed in living cells. Moreover, unlike our TPA to achieve an $S_{0} \rightarrow S_{1}\left(\pi-\pi^{*}\right)$ electronic transition in retinal, all these previous studies used 1PA excitation, which cannot be used to attain spatial specificity and deeper penetration in tissue. We demonstrated that even though the instantaneous TPA probability (Fig. 2d and Supplementary Fig. 7) and excitation pulse width are identical for both $\mathrm{PCH}$ and $\mathrm{NCH}$, the isomerization of all-trans retinal in the ChR2 protein environment is favoured with $\mathrm{PCH}$ due to the phase ordering of its spectral bandwidth. Furthermore, the ensuing state preparations modulated the total number of channel openings and hence, the light-evoked current, making this the first experimental evidence of quantum coherent control of biological function of a protein in its natural tissue environment.

We turned to existing theories of coherent control to search for a mechanism that might explain this observation. The pumpdump mechanism originally proposed by Tannor and Rice ${ }^{31-33}$ can explain how the PCH excitation pulse leads to enhancement of the $S_{1}$ population, and thus selectivity in photochemical reactions. After excitation, the wave packet on $S_{1}$ moves from higher to lower optical frequencies, and the dumping is more efficient for the NCH pulse. However, this mechanism is suitable only for strong field interactions at very high excitation power $^{3}$, where a molecule can potentially cycle many times during the duration of the excitation puls $\mathrm{e}^{33,34}$. This most likely is not the case here, as a relatively low excitation energy must be used for live tissue measurements. Our observations are possibly better explained with an alternative mechanism of excitation via wave packet motion ${ }^{2,3,34}$, which depends on the creation of vibrational coherences. For weakmoderate field transitions, the chirp does not directly affect the $S_{0}$ and $S_{1}$ populations, but rather the vibrational coherence within those populations on the electronic potential energy surfaces. The final state preparation is controlled, and a $\mathrm{PCH}$ pulse with its comb of subpulses ${ }^{34}$ favours formation of this resonant coherent condition during the lifetime of the femtosecond pulse by controlling the wave packet propagation, thus indirectly affecting the outcome of all-trans to 13-cis photoisomerization.

No study exists for the direct estimation of the degree of decoherence for ChR2 in a live tissue environment. Spectroscopic studies from other groups have demonstrated that in the photoexcitation of rhodopsin, an opsin system responsible for vision, the transition from the reactant photoexcited state to the ground state photoproduct is very fast $(50 \mathrm{fs})^{5,30}$ and vibrationally coherent. Even though this level of quantum coherent control is somewhat unexpected in complex membrane protein systems such as opsins in a living tissue environment, the source of the control very likely is the initial state preparation, which was directly controlled by the chirped light. Then, the field on-condition due to the 1,200 ms laser exposure was what created the control of the final state and the isomerization quantum yield ${ }^{35,36}$. The subsequent coherence was inconsequential due to the fact that more transfer to the ground state, or mixing with other vibronic levels with lower transmission probability through the conical intersection, will also produce a similar effect, independent of the decoherence timescales.

We demonstrated that it is possible to achieve quantum coherent control of ChR2, signifying that wave properties of matter can be detected for a channel protein in a live brain tissue environment. We chose an opsin for our experiment as it allowed for a straightforward measurement of functional output after photostimulation. However, it is noteworthy that quantum coherence control of protein function can arguably be extended to other light-sensitive proteins.
The degree of modulation for such a protein will depend on the timescales of photoisomerization (or other initiation reactions), thermal motion, and vibrational relaxation, but in essence, these experiments are the first demonstration of a more generalized physical phenomenon and, in principle, it should be possible to attain similar effects with other photosensitive proteins in living cells. Another significance of this work is that we achieved this coherent control of a channel protein using TPA, which can eventually be translated into living animals with a high degree of spatial selectivity in three dimensions. Additionally, we also report that the photo-activated cell function is controlled by pulse shaping, and this might be used to establish a new direction for optogenetics studies. Optogenetics has changed the way neuronal function is modulated in cells. The field has emerged from the neuroscience community, and focused mainly on developing engineered synthetic opsins for faster and more efficient stimulation of cells. Instead, it would be interesting to achieve the same goal by changing the light pulse shaping parameters. The key is that we have developed a novel control mechanism that can now be encoded into the complex time structure of ultrafast pulses to probe and modulate neuronal networks and, in particular, the overall interactions that enable the system to behave efficiently.

In other pulse-shaping applications outside of neuroscience, an adaptive feedback approach has been used in which feedback error signals between a measured and a desired output are fed to a learning or optimization algorithm to iteratively reduce the error $^{37-39}$. Such adaptive feedback control for the pulse-shaped light stimulus has been successfully used in laser-based coherently controlled chemistry $\mathrm{y}^{37,40}$, and has been increasingly popular in other applications ${ }^{39}$. An application for pulse shaping may be in closedloop optical control, where the current output spike pattern is compared to the desired spike pattern and a recursive algorithm (for example, genetic algorithm) is used to generate the feedback error signal to compute chirp values to produce output spike patterns closer to the desired output. Future investigations in our laboratory will explore the use of pulse shaping in this direction.

\section{Methods}

Methods, including statements of data availability and any associated accession codes and references, are available in the online version of this paper.

Received 12 September 2016; accepted 15 August 2017; published online 18 September 2017

\section{References}

1. Bardeen, C. J., Wang, Q. \& Shank, C. V. Femtosecond chirped pulse excitation of vibrational wave packets in LD690 and bacteriorhodopsin. J. Phys. Chem. A 102, 2759-2766 (1998).

2. Prokhorenko, V. I. et al. Coherent control of retinal isomerization in bacteriorhodopsin. Science 313, 1257-1261 (2006).

3. Prokhorenko, V. I., Nagy, A. M., Brown, L. S. \& Dwayne Miller, R. J. On the mechanism of weak-field coherent control of retinal isomerization in bacteriorhodopsin. Chem. Phys. 341, 296-309 (2007).

4. Prokhorenko, V. I., Halpin, A., Johnson, P. J., Miller, R. J. \& Brown, L. S. Coherent control of the isomerization of retinal in bacteriorhodopsin in the high intensity regime. J. Chem. Phys. 134, 085105 (2011).

5. Johnson, P. J. et al. Local vibrational coherences drive the primary photochemistry of vision. Nat. Chem. 7, 980-986 (2015).

6. Fenno, L., Yizhar, O. \& Deisseroth, K. The development and application of optogenetics. Annu. Rev. Neurosci. 34, 389-412 (2011).

7. Mattis, J. et al. Principles for applying optogenetic tools derived from direct comparative analysis of microbial opsins. Nat. Methods 9, 159-172 (2012).

8. Oron, D., Papagiakoumou, E., Anselmi, F. \& Emiliani, V. Two-photon optogenetics. Prog. Brain Res. 196, 119-143 (2012).

9. Schrodel, T., Prevedel, R., Aumayr, K., Zimmer, M. \& Vaziri, A. Brain-wide 3D imaging of neuronal activity in Caenorhabditis elegans with sculpted light. Nat. Methods 10, 1013-1020 (2013). 
10. Papagiakoumou, E. et al. Scanless two-photon excitation of channelrhodopsin-2. Nat. Methods 7, 848-854 (2010).

11. Andrasfalvy, B. K., Zemelman, B. V., Tang, J. \& Vaziri, A. Two-photon single-cell optogenetic control of neuronal activity by sculpted light. Proc. Natl Acad. Sci. USA 107, 11981-11986 (2010).

12. Hegemann, P., Gartner, W. \& Uhl, R. All-trans retinal constitutes the functional chromophore in Chlamydomonas rhodopsin. Biophys. J. 60, 1477-1489 (1991).

13. Schneider, F., Grimm, C. \& Hegemann, P. Biophysics of channelrhodopsin. Annu. Rev. Biophys. 44, 167-186 (2015).

14. Mathies, R. A., Cruz, C. H. B., Pollard, W. T. \& Shank, C. V. Direct observation of the femtosecond excited-state cis-trans isomerization in bacteriorhodopsin. Science 240, 777-779 (1988).

15. Bamann, C., Kirsch, T., Nagel, G. \& Bamberg, E. Spectral characteristics of the photocycle of channelrhodopsin-2 and its implication for channel function. J. Mol. Biol. 375, 686-694 (2008).

16. Scholz, F., Bamberg, E., Bamann, C. \& Wachtveitl, J. Tuning the primary reaction of channelrhodopsin-2 by imidazole, $\mathrm{pH}$, and site-specific mutations. Biophys. J. 102, 2649-2657 (2012)

17. Verhoefen, M. K. et al. The photocycle of channelrhodopsin-2: ultrafast reaction dynamics and subsequent reaction steps. Chemphyschem: Eur. J. Chem Phys. Phys. Chem. 11, 3113-3122 (2010).

18. Shapiro, M. \& Brumer, P. Quantum Control of Molecular Processes Second, Revised and Enlarged Edition edn (WILEY-VCH Verlag GmbH \& Co. $\mathrm{KGaA}, 2012$ ).

19. Dantus, M. \& Lozovoy, V. V. Experimental coherent laser control of physicochemical processes. Chem. Rev. 104, 1813-1859 (2004).

20. Treacy, E. B. Optical pulse compression with diffraction gratings. IEEE J. Quantum Electron. 5, 454-458 (1969).

21. Liu, Y., Tu, H., Benalcazar, W. A., Chaney, E. J. \& Boppart, S. A. Multimodal nonlinear microscopy by shaping a fiber supercontinuum from 900 to $1160 \mathrm{~nm}$. IEEE J. Sel. Top. Quantum Electron. 18, 1209-1214 (2012).

22. Kim, J. E., Tauber, M. J. \& Mathies, R. A. Analysis of the mode-specific excited-state energy distribution and wavelength-dependent photoreaction quantum yield in rhodopsin. Biophys. J. 84, 2492-2501 (2003).

23. Kim, J. E., Tauber, M. J. \& Mathies, R. A. Wavelength dependent cis-trans isomerization in vision. Biochemistry-Us 40, 13774-13778 (2001).

24. Pollard, W. T., Lee, S.-Y. \& Mathies, R. A. Wave packet theory of dynamic absorption spectra in femtosecond pump-probe experiments. J. Chem. Phys. 92, 4012-4029 (1990)

25. Wang, Q., Schoenlein, R. W., Peteanu, L. A., Mathies, R. A. \& Shank, C. V. Vibrationally coherent photochemistry in the femtosecond primary event of vision. Science 266, 422-424 (1994).

26. Hasson, K. C., Gai, F. \& Anfinrud, P. A. The photoisomerization of retinal in bacteriorhodospin: experimental evidence for a three-state model. Proc. Natl Acad. Sci. USA 93, 15124-15129 (1996).

27. González-Luque, R. et al. Computational evidence in favor of a two-state, two-mode model of the retinal chromophore photoisomerization. Proc. Natl Acad. Sci. USA 97, 9379-9384 (2000).

28. Koyabashi, T., Saito, T. \& Ohtani, H. Real-time spectroscopy of transition states in bacterorhodopsin during retinal isomerization. Nature 414, 531-534 (2001).

29. Hayashi, S., Tajkhorshid, E. \& Schulten, K. Molecular dynamics simulation of bacteriorhodopsin's photoisomerization using ab initio forces for the excited chromophore. Biophys. J. 85, 1440-1449 (2003).
30. Polli, D. et al. Conical intersection dynamics of the primary photoisomerization event in vision. Nature 467, 440-443 (2010).

31. Tannor, D. J. \& Rice, S. A. Control of selectivity of chemical reaction via control of wave packet evolution. J. Chem. Phys. 83, 5013-5018 (1985).

32. Tannor, D. J., Kosloff, R. \& Rice, S. A. Coherent pulse sequence induced control of selectivity of reactions: exact quantum mechanical calculations. J. Chem. Phys. 85, 5805-5820 (1986).

33. Cerullo, G., Bardeen, C. J., Wang, Q. \& Shank, C. V. HIgh-power femtosecond chirped pulse excitation of molecules in solution. Chem. Phys. Lett. 262, 362-368 (1996)

34. Prokhorenko, V. I., Nagy, A. M. \& Miller, R. J. Coherent control of the population transfer in complex solvated molecules at weak excitation. An experimental study. J. Chem. Phys. 122, 184502 (2005).

35. Dantus, M. Coherent nonlinear spectroscopy: from femtosecond dynamics to control. Annu. Rev. Phys. Chem. 52, 639-679 (2001).

36. Rosker, M. J., Dantus, M. \& Zewail, A. H. Femtosecond clocking of the chemical bond. Science 241, 1200-1202 (1988).

37. Assion, A. et al. Control of chemical reactions by feedback-optimized phase-shaped femtosecond laser pulses. Science 282, 919-922 (1998).

38. Baumert, T., Brixner, T., Seyfried, V., Strehle, M. \& Gerber, G. Femtosecond pulse shaping by an evolutionary algorithm with feedback. Appl. Phys. B 65, 779-782 (1997)

39. Weiner, A. M. Ultrafast optical pulse shaping: a tutorial review. Opt. Commun 284, 3669-3692 (2011).

40. Brixner, T., Damrauer, N. H., Niklaus, P. \& Gerber, G. Photoselective adaptive femtosecond quantum control in the liquid phase. Nature 414, 57-60 (2001).

\section{Acknowledgements}

We are grateful to J. Suarez, P. Pande, J. S. Rhodes and M. U. Gillette for their valuable comments, to S. You and Y.-Z. Liu for their assistance with the optics, and

M. H. W. Gruebele for careful reading of the manuscript. We thank E. Chaney for his assistance with the animal protocols, and D. Spillman Jr for his laboratory operations support. We also appreciate the collaborative efforts from Biophotonics Solutions, Inc., Michigan, USA. This research was supported in part by grants from the National Science Foundation, CBET 14-03660 and BRAIN EAGER CBET 14-50829, the National Institutes of Health, R01 EB023232, and from a seed grant from the Beckman Institute for Advanced Science and Technology. Additional information can be found at http://biophotonics.illinois.edu.

\section{Author contributions}

K.P., P.S. and S.A.B. designed experiments. E.D.A., H.T., K.P. and Y.Z. developed and maintained the optical set-up. K.P. and E.D.A. performed electrophysiology experiments with chirped pulse stimulation. K.P., P.S. and S.A.B. performed data analysis. The manuscript was written primarily by P.S. and S.A.B., with contributions from the other authors. S.A.B. obtained funding for this research.

\section{Additional information}

Supplementary information is available in the online version of the paper. Reprints and permissions information is available online at www.nature.com/reprints. Publisher's note: Springer Nature remains neutral with regard to jurisdictional claims in published maps and institutional affiliations. Correspondence and requests for materials should be addressed to S.A.B.

\section{Competing financial interests}

The authors declare no competing financial interests. 


\section{Methods}

Optics. The schematic for the optical set-up is shown in Supplementary Fig. 1a A $470 \mathrm{~nm}$ LED (M470F1, Thorlabs) was used for 1PA excitation of ChR2 (Supplementary Fig. 2b,d). After obtaining robust spiking and substantial peak current responses, the light source was switched to the output from the pulse-shaper set-up (Supplementary Fig. 1a), which delivered the tailored light pulses for two-photon absorption (TPA) by ChR2. To obtain instantaneous TPA and an ionic current response, the laser beam was not scanned for these experiments. The beam paths of the $470 \mathrm{~nm}$ light and shaped light were spatially overlapped under the microscope objective, and a flip mirror was used to switch between the two beams (Supplementary Fig. 1). A mode-locked ytterbium laser source (High-Q) was used to generate ultrashort NIR pulses (Supplementary Fig. 1c) which were subsequently coupled into a nonlinear supercontinuum generating PCF (NKT Photonics, cat. \# NL-1050-NEG-1) to spectrally broaden the pulses for shaping over $960-1,060 \mathrm{~nm}, \lambda_{\mathrm{c}}=1,010 \mathrm{~nm}$. The output of the PCF (Supplementary Fig. 1d) was coupled to the pulse-shaper unit (femtoJock, BioPhotonics Solutions). The pulse-shaper unit consisted of a diffraction grating that was used to spectrally and spatially separate the wavelengths. A curved mirror was employed to focus the beam onto a pixelated spatial light modulator (SLM) to modulate individual wavelengths. The SLM consisted of an array of liquid crystals to which an electric field may be applied to advance or retard the phase of individual spectral components within the pulses. The number of pixels in the SLM (128) was roughly equal to the spectral bandwidth of each pulse $(100 \mathrm{~nm})$. An electric field was generated in each pixel, which changed the orientation of the liquid crystals, and consequently their refractive index. In this way, the phase of the individual wavelengths was modulated. The second half of the pulse-shaper unit consisted of a second curved mirror that focused the spectrally shaped phase envelope onto a diffraction grating, and the recombined wavelength components constitute the output pulse that was directed to the slice tissue through a $60 \times$ objective (N.A. $=0.9$ ). The diameter of the spot size was $1.4 \mu \mathrm{m}$. For two-photon excitation, the average laser power was $13-19 \mathrm{~mW}$ (fluence: $5.4-8 \times 10^{16}$ photons $\mathrm{cm}^{-2}$ pulse $\left.{ }^{-1}\right)$. The pulse duration for each pulse was $\leq 350 \times 10^{-15} \mathrm{~s}$ (Supplementary Fig. 7). The laser light was 'on' (or consider that the shutter was open) for $1,200 \mathrm{~ms}$, which is denoted by the red bar in the figures.

The phase of the spectrally shaped or 'tailored' light is given by a polynomial function:

$$
\varphi(\omega)=\varphi_{0}+\varphi_{1}\left(\omega-\omega_{0}\right)+\left(\varphi_{2} / 2 !\right)\left(\omega-\omega_{0}\right)^{2}+\cdots
$$

where $\varphi(\omega)$ is the phase of the spectrally shaped pulse as a function of frequency $\omega$, and $\varphi_{0}, \varphi_{1}, \varphi_{2}$, are the increasing higher-order terms in this Taylor series expansion around the carrier frequency $\omega_{0}$. In the pulse-shaping software control module (BioPhotonics Solutions), the coefficients of the spectral phase function are given by $a 0-a 4$, and we have used this convention in our results.

The phases of the individual wavelengths of the spectrally broadened pulses out of the PCF have a specific phase relationship with each other that depends on the particular path taken by the pulses within the fibre and its properties. Using a multiphoton intrapulse interference phase scan (MIIPS), a previously determined 'phase compensation mask' was generated so that the phase of the spectrally filtered PCF output at the microscope objective was made uniform (that is, compensated). This was generated based on the second harmonic generated signal (SHG) of the PCF output at the focus of the high numerical aperture objective ${ }^{41}$ (see page 754-755 for details), using a commercial microscope detection unit (Biophotonics Solutions). The phase compensation mask compensated for the undetermined phase of the individual wavelengths and aligned all of the wavelengths to the same phase ${ }^{42}$. After phase compensation, the input pulse was compressed from $\sim 250 \mathrm{fs}$ to $\sim 30 \mathrm{fs}$. However, each femtosecond pulse contained the same number of photons and energy as the original uncompensated pulse. By introducing a linear chirp into the polynomial function above, the phase envelope of the tailored light was altered in a controlled and predetermined manner. The linear chirp coefficient $a 2$ was related to the pulse duration $\tau$ through $^{20,43}$ :

$$
\tau^{2}=\tau_{0}^{2}+\left[\frac{4(a 2) \ln 2}{\tau_{0}}\right]^{2}
$$

where $\tau$ is the pulse duration of the chirped laser pulse and $\tau_{0}$ is the chirp-free pulse duration (full-width at half-maximum, FWHM) of the TL pulse. Therefore, introduction of linear chirp again broadened the pulse, but the total energy per pulse remained unchanged.

SHG measurement. We measured an SHG signal (Fig. 2d) from an SHG crystal as an established reference method and control to demonstrate that, in the absence of any biological sample, no differences existed between PCH and NCH effects. The SHG measurement using an SHG crystal is a process afforded by our Multiphoton Intrapulse Interference Phase Scan (MIIPS). After the laser beam was coupled into the microscope and focused by the objective to a diffraction-limited point inside the crystal, the generated SHG signal at half of the laser wavelength was collected by a spectrometer. The total intensity of the SHG signal was recorded as the integrated intensity of the SHG signal across its full spectral width. We then ran the MIIPS procedure to maximize the SHG signal, and directed the compensation phase mask obtained by MIIPS on to the SLM. This resulted in the zero chirp (symmetric point) shown in Fig. $2 \mathrm{~d}$. We then varied the chirp of the laser pulses using the MIIPS (pulse shaping), and recorded the variation of the SHG intensity as a function of the chirp. The PCH and the $\mathrm{NCH}$ of the same $a 2$ value gave the same SHG intensity, as expected by nonlinear optical physics. This established a reference based on the SHG measurement that is a trivial demonstration of coherent control of a two-photon process by showing that varying the chirp would not break the symmetry of the SHG response for either PCH or $\mathrm{NCH}$. We then replaced the SHG crystal with a living neuron embedded in its native tissue environment, and recorded the quantitative neuron response (that is, evoked current) due to the TPA of ChR2 as a function of the chirp.

Electrophysiology. All experimental procedures were carried out in accordance with the National Institutes of Health guidelines and approved by the Institutional Animal Care and Use Committee at the University of Illinois at Urbana-Champaign. ChR2-expressing transgenic mice

(B6.Cg-Tg(Thy1-COP4/EYFP)9Gfng/J from Jackson Lab, postnatal age: $14-45$ days) were deeply anaesthetized with a mixture of ketamine $\left(100 \mathrm{mg} \mathrm{kg}^{-1}\right)$ and xylazine $\left(3 \mathrm{mg} \mathrm{kg}^{-1}\right)$ and decapitated. The brain was quickly removed and placed into cold, oxygenated slicing medium containing (in $\mathrm{mM}$ ): $2.5 \mathrm{KCl}, 10.0$ $\mathrm{MgCl}_{2}, 0.5 \mathrm{CaCl}_{2}, 1.25 \mathrm{NaH}_{2} \mathrm{PO}_{4}, 26.0 \mathrm{NaHCO}_{3}, 11.0$ glucose, and 234.0 sucrose gassed with $95 \% \mathrm{O}_{2}-5 \% \mathrm{CO}_{2}$ to a final $\mathrm{pH}$ of 7.4. In some cases (age > P25), the anaesthetized animal was transcardially perfused with the oxygenated slicing medium before decapitation and brain removal. Tissue slices ( $300 \mu \mathrm{m}$ thickness) were cut in the coronal plane, transferred to a holding chamber, and incubated for $\geq 1 \mathrm{~h}$ before recording. Individual slices were transferred to a submersion-type recording chamber on a modified microscope stage and continuously superfused with oxygenated physiological saline at room temperature. The physiological solution used in the experiments contained (in $\mathrm{mM}$ ): $126.0 \mathrm{NaCl}, 2.5 \mathrm{KCl}, 1.25$ $\mathrm{MgCl}_{2}, 2.0 \mathrm{CaCl}_{2}, 1.25 \mathrm{NaH}_{2} \mathrm{PO}_{4}, 26.0 \mathrm{NaHCO}_{3}$, and 10.0 glucose. This solution was gassed with $95 \% \mathrm{O}_{2} / 5 \% \mathrm{CO}_{2}$ to a final $\mathrm{pH}$ of 7.4 .

A Multiclamp 700B amplifier (Molecular Devices) was used in current and voltage clamp mode for the recordings. Intracellular recording micropipettes had tip resistances of 3-6 M $\Omega$ when filled with an intracellular solution containing (in mM): $117 \mathrm{~K}$-gluconate, $13 \mathrm{KCl}, 1.0 \mathrm{MgCl}_{2}, 0.07 \mathrm{CaCl}_{2}, 0.1 \mathrm{EGTA}, 10.0 \mathrm{HEPES}$, $2.0 \mathrm{Na}_{2}$-ATP, and $0.4 \mathrm{Na}$-GTP. The apparent input resistance was calculated from the linear slope of the voltage-current relationship near rest, obtained by applying constant-current pulses ranging from $-100 \mathrm{pA}$ to $+40 \mathrm{pA}$ (500 ms duration), and was maintained at $\leq 20 \mathrm{M} \Omega$ throughout the recording.

In current clamp recordings, an active bridge circuit was continuously adjusted to balance the drop in potential produced by passing current through the recording electrode. The apparent input resistance was calculated from the linear slope of the voltage-current relationship near rest, obtained by applying constant-current pulses ranging from $-100 \mathrm{pA}$ to $+40 \mathrm{pA}$ (500 ms duration). In voltage clamp recordings, currents were measured in the presence of tetrodotoxin (TTX: $0.5 \mu \mathrm{M}$, Sigma) to avoid spontaneous firing of action potentials. The data acquisition frequency was set at $10 \mathrm{kHz}$.

In voltage clamp experiments where current responses to negative and positive chirp coefficients were obtained from the same neuron (Fig. 2), the order of trials in 5 of 6 cells was $a 2=0$ (control), $a 2=$ negative coefficient, $a 2=$ positive coefficient and $a 2=0$ (recovery). In 1 of 6 cells, the order was $a 2=0$ (control), $a 2=$ positive coefficient, $a 2=$ negative coefficient and $a 2=0$ (recovery). There was a minimum interval of $45 \mathrm{~s}$ between the end of one trial and the beginning of the next so that the ChR2 photocycle from the previous activation was completely extinguished ${ }^{15}$.

Two-photon absorption (TPA) probability calculation. The probability of TPA is much smaller than that for single-photon excitation. It is nonlinear and increases with the square of photon intensity $I^{2}$. When working with a pulsed laser source of pulse width $\tau_{\mathrm{p}}$ and a focusing lens, TPA probability per pulse $\left(n_{\mathrm{a}}\right)$ can be defined as ${ }^{44}$ :

$$
n_{\mathrm{a}} \propto \frac{\sigma_{2} P_{\text {avg }}^{2}}{\tau_{\mathrm{p}} f_{\mathrm{p}}^{2}}\left(\frac{\mathrm{NA}^{2}}{2 \hbar c \lambda}\right)^{2}
$$

where $\sigma_{2}$ is the two-photon cross-section of the fluorophore at wavelength $\lambda, P_{\text {avg }}$ is the laser beam average power, NA is the numerical aperture of the focusing lens, $\hbar$ is the reduced Planck's constant, and $c$ is the speed of light. As peak power of the pulse, $P_{\text {peak }}$ is defined as:

$$
P_{\text {peak }}=\frac{P_{\text {avg }}}{f_{\mathrm{p}} \tau_{\mathrm{p}}}
$$


where $f_{\mathrm{p}}$ is the repetition rate of the laser source, equation (3) can be modified as:

$$
n_{\mathrm{a}} \propto \sigma_{2} P_{\text {peak }}^{2} \tau_{\mathrm{p}}
$$

for an experiment if all other parameters remain unchanged.

For comparative analysis, TPA probabilities per pulse (Supplementary Fig. 7) for chirped and other pulses were calculated using equations (2)-(5). Additionally, peak amplitude and FWHM were measured from experimentally generated pulse intensity profiles at different $a 2$ values. These values were used for a parallel estimation of TPA. An average of theoretically calculated and experimentally estimated TPA is plotted in Supplementary Fig. $7 \mathrm{~b}$ for comparison with measured current values with different $\mathrm{PCH}$ and $\mathrm{NCH}$ pulses. (The evoked current is proportional to TPA). Please note that the authors understand that for a chirped pulse, TPA probability cannot be calculated accurately using equation (5) due to the combed structure embedded in the temporal profile of the pulse (Supplementary Fig. 7a). Actual TPA probability of a chirped pulse will be less than what we estimated with equation (5), so our conclusions about achieving higher TPA efficiency with chirping, at constant pulse peak power, still hold true.

Electrophysiology data analyses. Data was analysed using pCLAMP 10/Clampfit software (Molecular Devices). Voltage clamp data was low-pass-filtered at $150 \mathrm{~Hz}$ for analysis. The baseline data was taken as the average baseline current from $40 \mathrm{~ms}$ of data just before the start of two-photon stimulation and the peak current response was taken as the most negative current value after stimulation. The steady-state current was taken as the average of $40 \mathrm{~ms}$ data in the flat part of the response towards the end of the 1,200 ms stimulation. All voltage clamp data presented are negative inward currents.
The initial activation slope was calculated as $\mathrm{pA} \mathrm{ms} \mathrm{m}^{-1}$ from the initial $3 \mathrm{~ms}$ of current deflection immediately after two-photon activation. To compute this, the inward current obtained within $3 \mathrm{~ms}$ of laser activation was measured, and the current value thus obtained was then divided by 3 to obtain the initial activation slope.

Statistics. All data are presented as mean \pm s.e.m. Statistical analyses used the two-sample $t$-test and, when appropriate, a paired $t$-test was used (Figs 1 and 2 and Supplementary Figs 3-5 and 7). $p$-values $<0.05$ were considered to be statistically significant.

Data availability. The data that support the plots within this paper and other findings of this study are available from the corresponding author upon request.

\section{References}

41. Xu, B., Gunn, J. M., Dela Cruz, J. M., Lozovoy, V. V. \& Dantus, M. Quantitative investigation of the multiphoton intrapulse interference phase scan method for simultaneous phase measurement and compensation of femtosecond laser pulses. J. Opt. Soc. Am. B 23, 750-759 (2006)

42. Tu, H., Liu, Y., Turchinovich, D. \& Boppart, S. A. Compression of fiber supercontinuum pulses to the Fourier-limit in a high-numerical-aperture focus. Opt. Lett. 36, 2315-2317 (2011).

43. Siegman, A. A. Lasers (University Science Books, 1986).

44. Denk, W., Strickler, J. H. \& Webb, W. W. Two-photon laser scanning fluorescence microscopy. Science 248, 73-76 (1990). 